Purdue University

Purdue e-Pubs

2015

\title{
An Explicit Conditioning Method for Image Reconstruction in Electrical Capacitance Tomography
}

\author{
S. H. Taylor \\ Purdue University \\ S V. Garimella \\ Purdue University, sureshg@purdue.edu
}

Follow this and additional works at: http://docs.lib.purdue.edu/coolingpubs

Taylor, S. H. and Garimella, S V., "An Explicit Conditioning Method for Image Reconstruction in Electrical Capacitance Tomography" (2015). CTRC Research Publications. Paper 277.

http://dx.doi.org/dx.doi.org/10.1016/j.flowmeasinst.2015.10.009

This document has been made available through Purdue e-Pubs, a service of the Purdue University Libraries. Please contact epubs@purdue.edu for additional information. 


\title{
An Explicit Conditioning Method for Image Reconstruction in Electrical Capacitance Tomography ${ }^{1}$
}

\author{
Stephen H. Taylor and Suresh V. Garimella ${ }^{2}$ \\ Cooling Technologies Research Center, an NSF I/UCRC \\ School of Mechanical Engineering \\ Purdue University \\ West Lafayette, IN 47907 USA
}

\begin{abstract}
A new electrical capacitance tomography (ECT) image reconstruction method, termed Sensitivity Factor Regularization (SFR), is developed. The SFR method provides an explicit formulation for solving the image reconstruction problem that performs better than other explicit methods, such as linear back-projection and Tikhonov regularization, while providing the same computational efficiency. The computational ease of the SFR method renders it an attractive option for ECT where real-time imaging is required and theoretical statistical evaluation of proposed electrode configurations may readily be performed. A statistical study is conducted using SFR image reconstructions for investigating the impact of electrode density on image quality for a symmetric ECT system characterizing a square cross-section. A larger number of smaller electrodes allows more data to be gathered for use in image reconstruction, but degrades signal-to-noise ratio in the measurements. The statistical study using SFR clearly identifies a theoretical optimum electrode density that minimizes reconstructed image error for a given level of measurement noise.
\end{abstract}

Keywords: image reconstruction, Sensitivity Factor Regularization (SFR), capacitance tomography, ECT design, electrode optimization 


\section{Introduction}

Diagnostic imaging of phase distributions in pipe flows is useful in various industrial settings. Although intrusive techniques may be employed [1,2], electrical capacitance tomography (ECT) provides a physically simple and nonintrusive method for imaging dielectric media. In ECT, electrodes are arranged around a domain of interest which contains an unknown distribution of multiple dielectric materials or material phases with significant contrast in electrical permittivity. A maximum of $M=K(K$ 1)/2 independent capacitance measurements are taken using unique electrode pairs, where $\mathrm{K}$ is the number of electrodes used. Each measurement represents an integrated value determined by the unique pattern of spatial sensitivity to the distribution of permittivity in the domain. The inverse problem of reconstructing the permittivity field (and thus, the distribution of materials or phases) is then solved using a suitable algorithm for mapping a vector of $\mathrm{M}$ known measurements to a vector of $\mathrm{N}$ unknown pixel values.

A canonical ECT problem is the reconstruction of a binary image representing a two-phase material distribution within a cross-section of a pipe, usually using between 8 and 16 electrodes [3, 4, 5, $6,7,8,9,10]$. Many options are available for solving the inverse problem, all characterized by a tradeoff between image quality and computation time, as will be quantitatively demonstrated in this work. A tradeoff also exists with respect to system design: Increasing the number of electrodes provides increased information from which to construct the image; however, the simultaneous decrease required of the electrode size results in poorer signal-to-noise ratio, making measurements more uncertain [11].

This work develops a new explicit image reconstruction method for capacitance tomography that yields solutions that are more accurate than other explicit approaches, but without increasing the computational cost. The image reconstruction method, termed Sensitivity Factor Regularization (SFR), suppresses false artifacts near electrode surfaces and provides improved image segmentation over the linear back-projection and Tikhonov formulations, making it an attractive option for ECT where real-time imaging is required. The accuracy and computational ease of the method makes it an ideal reconstruction algorithm for performing statistical evaluation and optimization of theoretical ECT systems. In this work, 
the SFR method is used to conduct a theoretical study of electrode design for a square, 2D domain of interest. As the electrode size is progressively reduced to accommodate a larger number of electrodes in the ECT system, the merit of the system is evaluated statistically via SFR reconstructions of a set of test images. It is shown that for an ECT system with finite measurement noise level, the optimum electrode size (and thus, electrode density) may be identified with respect to an $\mathrm{L}_{2}$ norm image error.

\begin{tabular}{|c|c|c|c|}
\hline \multicolumn{2}{|c|}{ Nomenclature } & \multirow{2}{*}{$\begin{array}{l}V \\
v\end{array}$} & \multirow{2}{*}{$\begin{array}{l}\text { voltage } \\
\text { volume }\end{array}$} \\
\hline$C$ & capacitance & & \\
\hline$C^{*}$ & normalized capacitance, $C^{*}=C / L \varepsilon_{o}$ & $v^{*}$ & normalized volume, $v^{*}=v / L^{3}$ \\
\hline D & diagonal matrix & & \\
\hline$E$ & image error & $\mathrm{x}$ & $\mathrm{x}$ direction \\
\hline $\mathbf{E}$ & electric field & $x$ & vector of sensitivity factors \\
\hline $\mathbf{E}^{*}$ & normalized electric field, & $\mathrm{y}$ & y direction \\
\hline \multicolumn{2}{|c|}{$\mathbf{E}^{*}=L \mathbf{E} /\left(V_{h i g h}-V_{l o w}\right)$} & \multicolumn{2}{|c|}{ Greek Symbols } \\
\hline $\mathbf{I}$ & identity matrix & $\beta$ & vector of scaling coefficients \\
\hline $\mathrm{K}$ & number of electrodes & $\delta$ & regularization parameter \\
\hline$L$ & system length & $\varepsilon$ & permittivity \\
\hline M & number of measurements/electrode pairs & $\varepsilon_{o}$ & permittivity of free space \\
\hline $\mathrm{N}$ & number of mesh cells/pixels & $\varepsilon_{r}$ & relative permittivity, $\varepsilon_{r}=\varepsilon / \varepsilon_{o}$ \\
\hline $\mathbf{n}$ & unit normal vector & $\chi$ & normalized $\mathrm{x}, \chi=x / L$ \\
\hline $\mathbf{S}$ & sensitivity matrix & $\eta$ & normalized y, $\eta=y / L$ \\
\hline$\hat{\mathbf{S}}$ & augmented sensitivity matrix & $\varphi$ & normalized electric potential, \\
\hline$t$ & computation time & & $\varphi=\left(V-V_{\text {low }}\right) /\left(V_{\text {high }}-V_{\text {low }}\right)$ \\
\hline$u^{*}$ & normalized noise uncertainty & & \\
\hline & & $\mu$ & normalized measurement vector \\
\hline
\end{tabular}




$\begin{array}{llll}\sigma & \text { normalized length } & \text { clip } & \text { with min of } 0 \text { and max of } 1 \\ \xi & \text { image vector } & \text { img } & \text { image } \\ \omega & \text { local subdomain } & \text { map } & \text { vector mapping }\end{array}$

\section{Subscripts}

low with $\varepsilon_{\mathrm{r}}$ at low value

high with $\varepsilon_{\mathrm{r}}$ at high value
Superscripts

* normalized

$\wedge \quad$ pertaining to the SFR method

Individual matrix and vector entries are written with the matrix or vector name in non-bold type with identifying subscripts, e.g., $\mathbf{S}_{m, n}$ indicates the m,n component of $\mathbf{S}$.

\section{ECT System Modeling}

\subsection{Simulation Model}

A two-dimensional ECT system is considered, where the material under test (MUT) with a nominally high dielectric permittivity resides in a square domain of side length, $L$, with electrodes distributed around the perimeter. Either 4, 8, 12, or 16 electrodes may be included in the system as indicated in Figure 1. In each case, electrode size is chosen to be as large as possible while leaving two cell lengths of space between each electrode along the boundary. A finite-element model is used to solve for electric field in the square domain of the capacitance tomography system, and is composed of a regular mesh of $48 \times 48$ cells. 


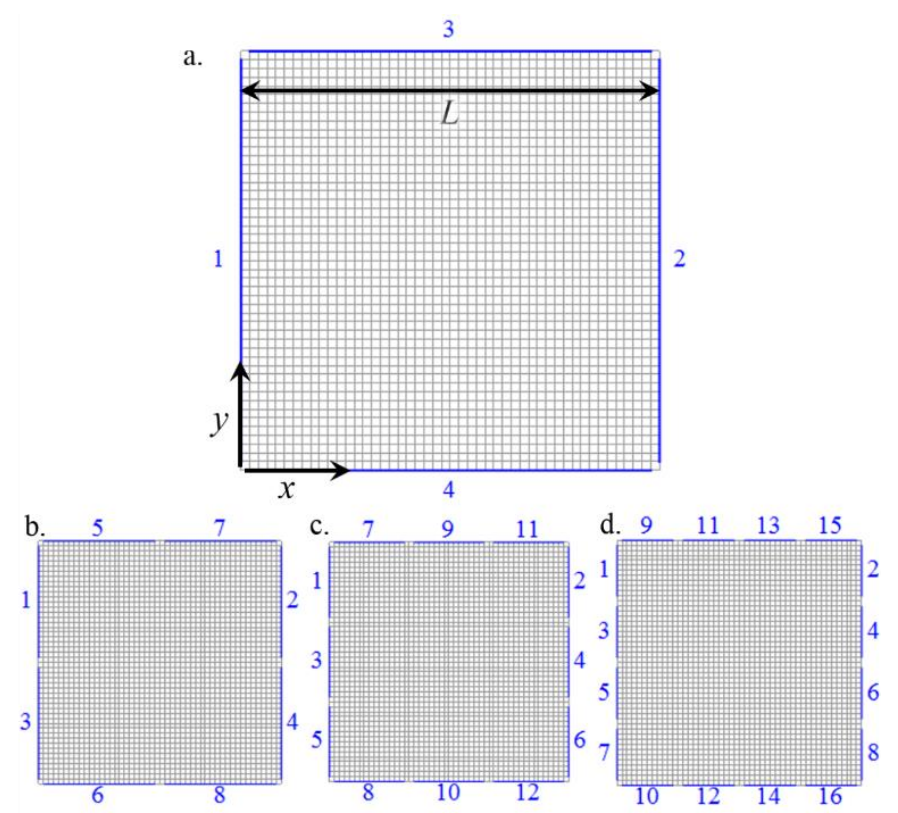

Figure 1. Domain and mesh for candidate tomography systems with (a) 4 electrodes, (b) 8 electrodes, (c) 12 electrodes, and (d) 16 electrodes.

The electrostatic field generated when obtaining capacitance measurements is governed by the divergence of the electric displacement field,

$$
\nabla \cdot\left(\varepsilon_{r} \nabla \varphi\right)=0
$$

When a measurement is simulated, a sender electrode is assigned a high constant potential, $\varphi=1$, while all other electrodes are assigned a low potential, $\varphi=0$. The portions of the boundaries of the domain that lie between electrodes have a zero flux condition. Displacement flux into any electrode assigned a low potential (receiver) may be used to determine the capacitance between that electrode and the highpotential electrode according to

$$
C^{*}=\underset{r e c e i v e r}{f_{r}} \nabla \varphi \cdot \mathbf{n} d \sigma
$$

In this way, a single simulation for electrode potential may be used to obtain K-1 capacitance measurements. It can be shown that capacitance of a given electrode pair is independent of which electrode plays the role of sender, the permittivity field $\varepsilon(x, y)$, and the presence of other fixed-potential 
bodies. Thus, a maximum of (K-1)K/2 independent measurements exist for any system, which may be characterized via K electric field solutions.

In this work, binary permittivity fields are considered, with regions of low permittivity set against a background of high permittivity. This corresponds to the common application of imaging voided regions, such as bubbles or voided cavities, existing in a highly dielectric MUT.

\subsection{Linearized Model}

Image reconstruction is performed using a linear model which approximates the causal relationship between a change in the permittivity of a mesh cell and a change in the capacitance of an electrode pair. The set of such relationships for all mesh cells is defined as the sensitivity distribution of the pair. The most common methodology for constructing the set of sensitivity distributions for all electrode pairs is to simulate perturbations in the electric field in an exhaustive fashion. A total of $\mathrm{N}$ complete measurement sets are simulated, where the $n^{\text {th }}$ case uses the same value for $\varepsilon_{\mathrm{r}}$ in all mesh cells except for cell $n$, which is perturbed by $\Delta \varepsilon_{\mathrm{r}}$. The relative impact of each cell on a simulated measurement is recorded under either a parallel assumption [3, 12], a series assumption [5], or a combination of both [8, 13]. While the computational expense of this technique is feasible for $2 \mathrm{D}$ systems, it is prohibitive for large 3D meshes [14]. Successful alternatives for calculating the sensitivity distribution have been developed based on electric field lines $[10,14,15]$.

Lucas et al. [16] showed that the first-order sensitivity of a capacitance measurement of an $i-j$ electrode pair to a change in capacitance of some sub-volume $\omega$, residing in the electric field may be expressed as

$$
\Delta C_{i, j}^{*} \cong \int_{\omega} \Delta \varepsilon_{r}\left(\mathbf{E}_{i, j}^{*} \cdot \mathbf{E}_{j, i}^{*}\right) d v^{*}
$$

where the subscript ' $i, j$ ' represents electrode $i$ as sender and electrode $j$ as receiver, with $\mathbf{E}^{*}$ and $\mathrm{v}^{*}$ are normalized electric field and volume, respectively. Equation 3 may be adapted to the finite element grid to define the sensitivity distributions. The raw discretized approximation of the change in measurement $m$, yielded by electrode pair $i$-j, due to a permittivity change in cell $n$ is defined as, 


$$
\Delta C_{m, n}^{*} \cong \Delta \varepsilon_{r, n}\left(\mathbf{E}_{i, j}^{*} \cdot \mathbf{E}_{j, i}^{*}\right)_{n} v_{n}^{*}
$$

Considerable computation may be saved by using Equation 4 instead of the exhaustive technique, allowing for the sensitivity distribution to be calculated with data from $K$ simulations instead of $N(K-1)$ simulations. In this work, the sensitivity matrix is generated using Equation 4, with electric fields solved using a uniform value of $\varepsilon_{\text {high }}$. Traditionally, capacitance measurements are normalized between 0 and 1 [11]. However, in this work, simulated measurements are referenced to individual datum values as

$$
\mu_{m}=\left(C_{m}^{*}\right)-\left(C_{m}^{*}\right)_{l o w}
$$

Using Equation 4, the referenced capacitance change of a pair may be represented approximately as

$$
\mu_{m} \cong \sum_{n=1}^{N} \xi_{n}\left(\varepsilon_{r, h i g h}-\varepsilon_{r, l o w}\right)\left(\mathbf{E}_{i, j}^{*} \cdot \mathbf{E}_{j, i}^{*}\right)_{n} v_{n}^{*}
$$

where $\xi_{n}$ takes on a value of 0 corresponding to $\varepsilon_{\text {low }}$ and a value of 1 corresponding to $\varepsilon_{\text {high }}$ based on the permittivity distribution in the domain. Figure 2 shows the difference between direct simulation (Equation 5) and the approximation (Equation 6) with measurements sorted by magnitude.

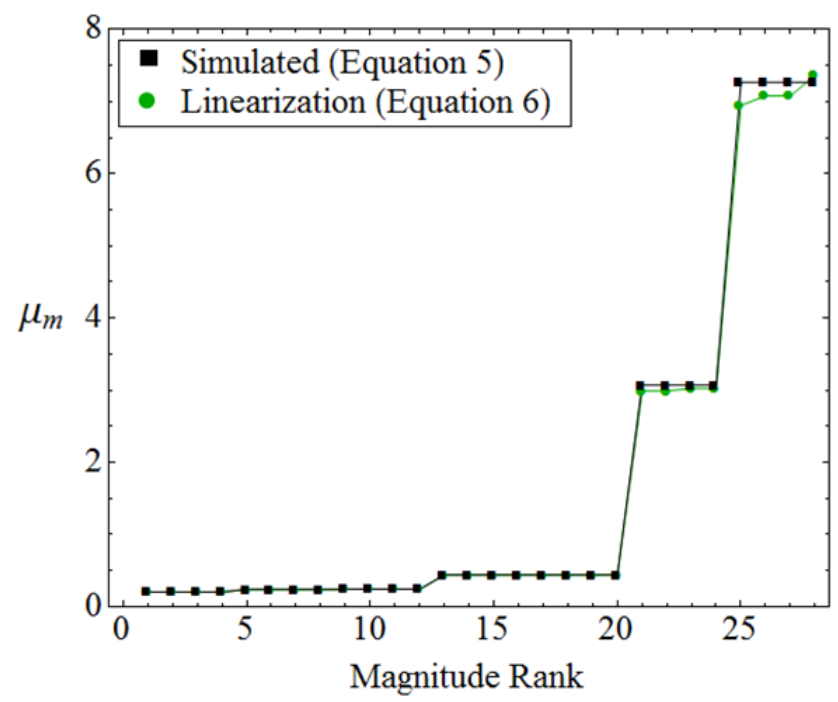

Figure 2. Referenced capacitance values obtained for the 8-electrode system obtained by direct simulation and by the linear approximation before the use of scaling factors, $\boldsymbol{\beta}$. Electric fields in Equation 6 calculated at $\varepsilon_{h i g h}$. 
The approximation is most accurate for measurements with small capacitance changes from the $\varepsilon_{\text {low }}$ case to the $\varepsilon_{\text {high }}$ case. In this work, scaling coefficients $\boldsymbol{\beta}$ are proposed, which ensure that the linearized model produces the same values of $\boldsymbol{\mu}$ obtained through the simulation model for the case when $\varepsilon(x, y, z)=\varepsilon_{\text {high }}$. The scaling factors are calculated as

$$
\beta_{m}=\frac{\left(C_{m}^{*}\right)_{h i g h}-\left(C_{m}^{*}\right)_{l o w}}{\sum_{n=1}^{N}\left(\varepsilon_{r, h i g h}-\varepsilon_{r, \text { low }}\right)\left(\mathbf{E}_{i, j}^{*} \cdot \mathbf{E}_{j, i}^{*}\right)_{n} v_{n}^{*}}
$$

The scaled approximate change in measurement $m$, yielded by electrode pair $i$-j, due to a permittivity change in cell $n$ is defined as

$$
\mathrm{S}_{m, n}=\beta_{m} \Delta C_{m, n}^{*}
$$

The $m \times n$ matrix $\mathbf{S}$ is known as the sensitivity matrix, representing a mapping between the vector spaces $\mathbf{R}^{\mathbf{m}}$ and $\mathbf{R}^{\mathbf{n}}$. Each row of $\mathbf{S}$ contains a discretized sensitivity distribution. Selected sensitivity distributions are illustrated in Figure 3. Each sensitivity distribution may be described as a tunnel of positive sensitivity between the two electrodes of the pair in a background of low-magnitude negative sensitivity. Note that measurements are far more sensitive to regions near electrodes than the central region of the domain. The linearized forward model is written as

$$
\mathbf{S} \xi=\mu
$$

with the image vector $\boldsymbol{\xi}$ representing pixel values. The inverse problem is to determine $\boldsymbol{\xi}$ from a known $\mathbf{S}$ and $\boldsymbol{\mu}$. 


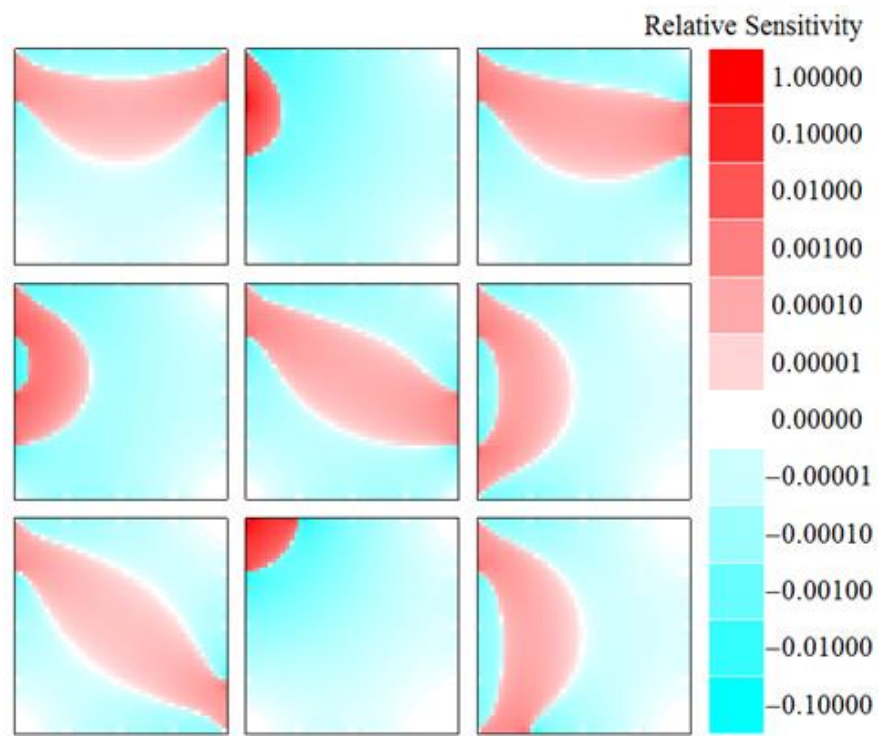

Figure 3. Selected sensitivity distributions from the 16-electrode design. Tunnels of positive sensitivity (red) reside within a background of negative sensitivity (blue).

\section{Reconstruction using Sensitivity Factor Regularization}

\subsection{Review of Image Reconstruction Algorithms}

Many methods have been investigated for obtaining the image vector, $\boldsymbol{\xi}$, from Equation 9. Iterative image reconstruction algorithms are widely used in the literature. The Landwebber iteration is chosen when computation time may be spent reconstructing image frames offline $[8,12,17]$. Basic Landwebber iteration is equivalent to a method of steepest descent using $\mathrm{N}$ variables and a fixed, small step size instead of a minimizing line search. Both convergence and binarization are enhanced by clipping the solution after each step, i.e. redefining any values greater than 1 as 1 and values less than 0 as 0 [11]. Other gradient-based methods include the algebraic reconstruction technique (ART) and the simultaneous iterative reconstruction technique (SIRT) [11]. Alternatively, the problem may be cast as a conditioned optimization problem, with examples including a least-squares regularization [18] or a total variation regularization (TV) $[19,20]$. Total variation regularization produces reconstructions characterized by coarse image segmentation, making it an attractive option when solutions are known to be binary. When using iterative algorithms, the sensitivity matrix may be updated at intervals by 
simulating measurements of the current estimated image [10], but at significant computational cost. Even if a constant sensitivity matrix is used, many iterations are usually required to converge to the final image.

When fast reconstruction is required for real-time monitoring, explicit or single-step algorithms are usually needed. Linear back projection (LBP) is a very computationally simple method, requiring only matrix multiplication of a renormalized, transposed version of the sensitivity matrix with the measurement vector $\boldsymbol{\mu}$ [12]. Other explicit image reconstruction methods include singular value decomposition (SVD) and Tikhonov regularization, which is equivalent to a filtered version of SVD [11]. Tikhonov regularization requires solving the system as

$$
\xi=\left(\mathbf{S}^{\mathrm{T}} \mathbf{S}+\delta \mathbf{I}\right)^{-1} \mathbf{S}^{\mathrm{T}} \boldsymbol{\mu}
$$

where $\delta$ is empirically chosen to ensure invertibility. In general, explicit techniques utilize a pseudoinverse matrix which may be stored a priori, trading some fidelity for large gains in computational efficiency.

In the present work, a new explicit image reconstruction technique, Sensitivity Factor Regularization (SFR), is proposed for fast image reconstruction which delivers better results than linear back projection or Tikhonov regularization.

\subsection{Unconditioned Sensitivity Factor Regularization (USFR)}

The concept underlying sensitivity factor regularization is that a vector $\boldsymbol{x}$ of $\mathrm{M}$ unknown sensitivity factors is defined, with each sensitivity factor corresponding to the sensitivity distribution of an electrode pair. These $\mathrm{M}$ unknown variables allow for $\mathrm{N}$ new constraints to be imposed on the system. The constraints take the form

$$
\xi_{n}=\frac{\sum_{m=1}^{M} x_{m} \mathrm{~S}_{m, n}}{\sum_{m=1}^{M} \mathrm{~S}_{m, n}}
$$

Equation 11 requires that the pixel value of each cell be expressed as a weighted average of the sensitivity factors of all sensitivity distributions, with weighting determined by the magnitudes of the sensitivity 
distributions at the particular cell. With the new constraints, the system contains $\mathrm{M}+\mathrm{N}$ variables and $\mathrm{M}+\mathrm{N}$ constraints, resulting in a fully constrained problem. The new system resulting from the application of Equation 11 may be expressed as an augmented matrix $\hat{\mathbf{S}}$, and augmented vectors $\hat{\boldsymbol{\xi}}$ and $\hat{\boldsymbol{\mu}}$, as

$$
\hat{\mathbf{S}} \hat{\xi}=\hat{\boldsymbol{\mu}} \text {, with } \hat{\mathbf{S}}=\left(\begin{array}{cc}
\mathbf{S}^{\mathbf{T}} & -\mathbf{D}_{\mathbf{S}} \\
\mathbf{0} & \mathbf{S}
\end{array}\right), \hat{\boldsymbol{\xi}}=\left(\begin{array}{l}
\boldsymbol{x} \\
\boldsymbol{\xi}
\end{array}\right), \hat{\boldsymbol{\mu}}=\left(\begin{array}{l}
\mathbf{0} \\
\boldsymbol{\mu}
\end{array}\right)
$$

The matrix $\mathbf{D}_{\mathbf{S}}$ is an $\mathbf{N} \times \mathrm{N}$ diagonal matrix with nonzero entries composed of the column sums of $\mathbf{S}$. Equation 12 may be solved directly, which we refer to as unconditioned sensitivity factor regularization (USFR).

When all rows of $\mathbf{S}$ are independent, then the first M columns of $\hat{\mathbf{S}}$ constitute a linearly independent set. If each column of $\mathbf{S}$ contains at least one nonzero entry, then the diagonal entries of $\mathbf{D}_{\mathbf{S}}$ are all nonzero, which guarantees that columns $\mathrm{M}+1$ through $\mathrm{M}+\mathrm{N}$ of $\hat{\mathbf{S}}$ form a linearly independent set. Finally, because each of the columns $\mathrm{M}+1$ through $\mathrm{M}+\mathrm{N}$ of $\hat{\mathbf{S}}$ contain at least one nonzero entry within rows $\mathrm{N}+1$ through $\mathrm{N}+\mathrm{M}$, (where all entries of columns 1 through $\mathrm{M}$ of $\hat{\mathbf{S}}$ are zero), each column vector in columns $\mathrm{M}+1$ through $\mathrm{M}+\mathrm{N}$ of $\hat{\mathbf{S}}$ is independent of columns 1 through $\mathrm{M}$. Thus, all column vectors are independent, and the system, $\hat{\mathbf{S}}$, is guaranteed to be of full rank $\mathrm{M}+\mathrm{N}$.

The full rank nature of $\hat{\mathbf{S}}$ suggests that no additional conditioning is required for solving the image reconstruction problem, making USFR an independently viable solution technique. Because USFR requires no design-specific, empirically determined conditioning parameters, significant computation may be saved when performing statistical studies of theoretical performance of many potential electrode designs. Image reconstructions with $\hat{\mathbf{S}}^{-1} \hat{\boldsymbol{\mu}}=\hat{\boldsymbol{\xi}}$ for the 8-electrode system are shown in Figure 4. The USFR method captures gross features of the images, although spurious artifacts far from electrode walls may still appear (Figure 4b) and small features may be lost (Figure 4e). 


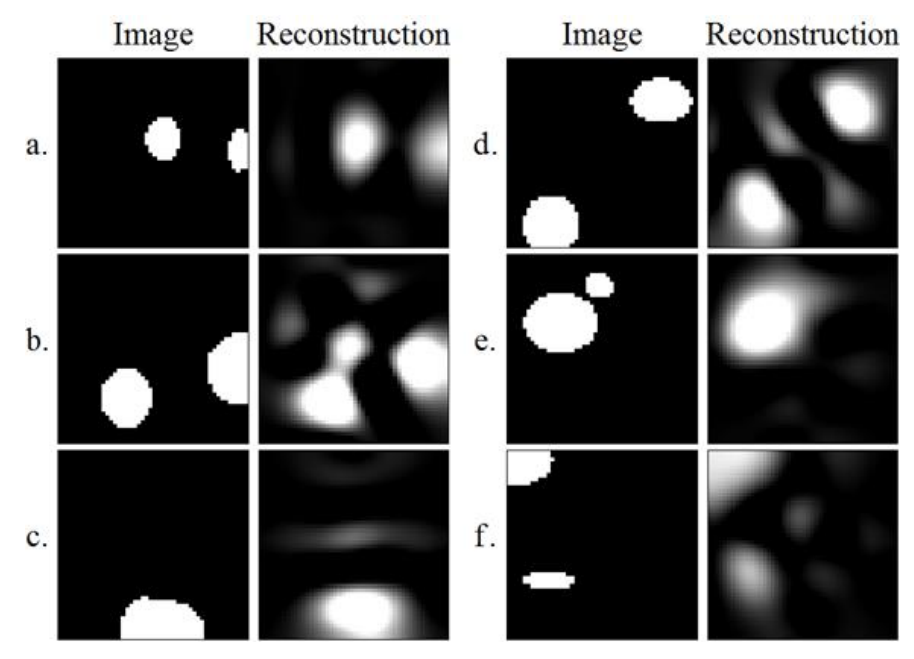

Figure 4. Image reconstructions for the 8-electrode system using the USFR method, $\hat{\mathbf{S}}^{-1} \hat{\boldsymbol{\mu}}=\hat{\boldsymbol{\xi}}$.

\subsection{Generalized sensitivity factor regularization (SFR)}

Although $\hat{\mathbf{S}}$ may be of full rank $\mathrm{M}+\mathrm{N}$ in principle, it may be poorly conditioned. The $\hat{\mathbf{S}}$ matrix corresponding to the 16-electrode system is observed to be too ill-conditioned to invert effectively. When this is the case, Tikhonov conditioning may be applied, yielding

$$
\hat{\boldsymbol{\xi}}=\left(\hat{\mathbf{S}}^{\mathrm{T}} \hat{\mathbf{S}}+\hat{\delta} \mathbf{I}\right)^{-1} \hat{\mathbf{S}}^{\mathrm{T}} \hat{\boldsymbol{\mu}}
$$

We refer to Equation 13 as the generalized SFR method, with USFR corresponding to the case where $\hat{\delta}$ $=0$. Tikhonov conditioning may also suppress noise artifacts and thus improve accuracy of the reconstructions. For consistency in the present study, all image reconstructions (with the exception of Figure 4) are performed with a value of $\hat{\delta}$ which is optimized separately for each candidate ECT system (see Section 4.1). The value of $\delta$ used in Equation 10 for standard Tikhonov regularization is similarly optimized for purposes of comparison.

\subsection{Benchmark comparisons with SFR}

In Figure 5, the SFR method is compared to well-known image reconstruction methods for ECT, both explicit and iterative. Values for $\mathrm{L}_{2}$ norm image error, given by 


$$
E_{\text {img }}=\left\|\xi_{\text {clip }}-\left(\frac{\boldsymbol{\varepsilon}-\varepsilon_{\text {low }}}{\varepsilon_{\text {high }}-\varepsilon_{\text {low }}}\right)\right\|_{2} \text { with } \xi_{\text {clip }, n}=\left\{\begin{array}{cc}
1 & \xi_{n}>1 \\
\xi_{n} & 0 \leq \xi_{n} \leq 1 \\
0 & \xi_{n}<0
\end{array}\right.
$$

are provided in Figure 5 along with computation time $t$ on a Core ${ }^{\mathrm{TM}}$ i7 $2 \mathrm{GHz}$ processor and $12 \mathrm{~GB}$ of memory using Wolfram Mathematica [21]. Computation times are below the runtime resolution limit for all of the explicit methods. Computation times for the iterative methods should be interpreted as qualitative, as these strongly depend on user choices for parameters such as step sizes and convergence criteria. In the case of TV regularization, several algorithms exist to accomplish the same regularization objective. The continuation algorithm [22] was used for TV in Figure 5, while other options include a Broyden Fletcher Goldfarb Shanno (BFGS)-based technique [22] and the alternating direction method of multipliers (ADMM) algorithm [23]. Image binarization is better for the iterative algorithms than for the explicit algorithms. Among the explicit algorithms, linear back projection performs very poorly at image segmentation. Tikhonov regularization provides sufficient resolution to recognize the number of objects in the image, but suffers from spurious artifacts near walls. The SFR method suppresses the false artifacts and provides discernible improvement in image binarization; suppressing artifacts is the primary reason for the dramatic reduction of overall image error compared to the other explicit methods. Although binarization of the SFR reconstructions is inferior to that of the iterative algorithms, overall image error is comparable. As seen in Figure 5, this accuracy is provided at a small fraction of the computation time required for any of the iterative algorithms. 


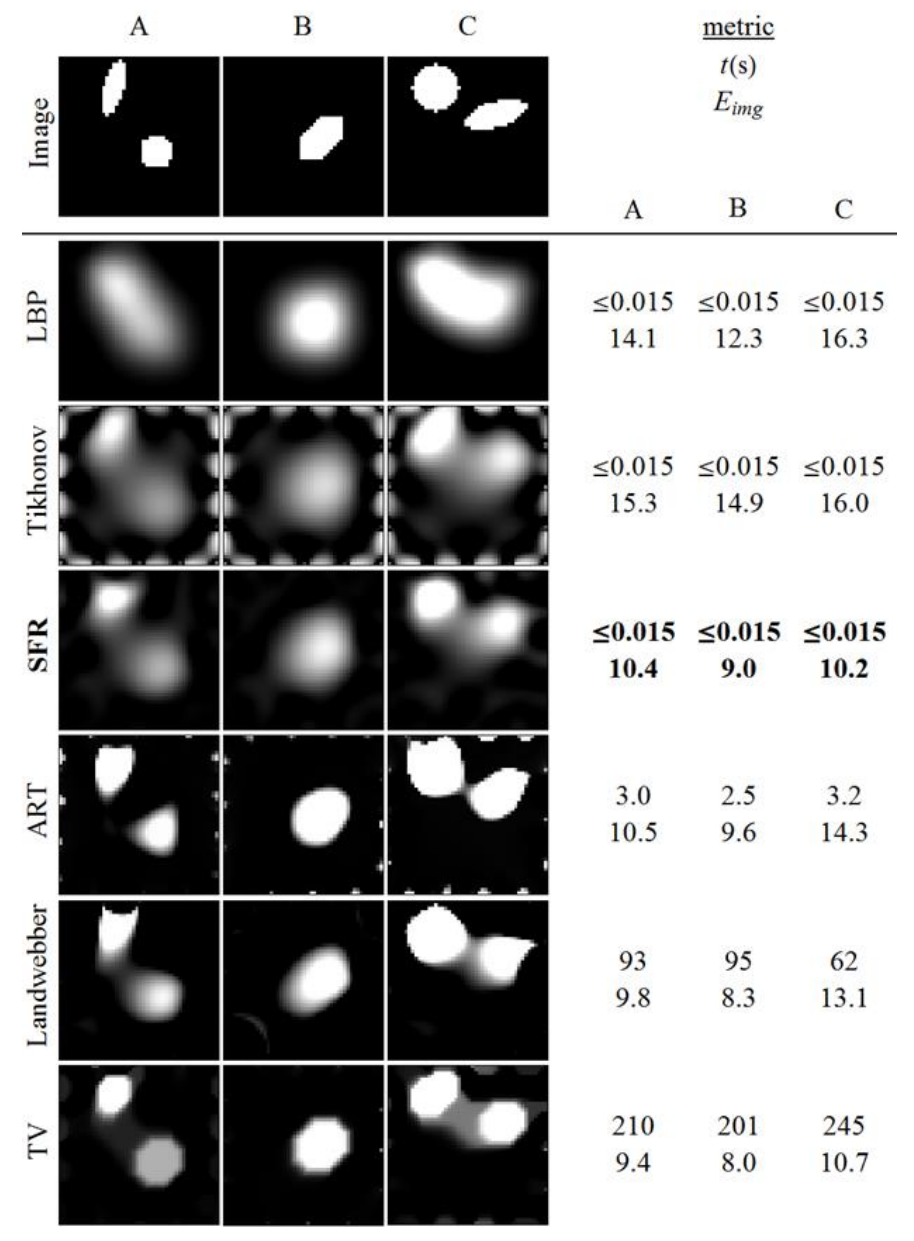

Figure 5. Comparative study of image reconstructions using various explicit and iterative methods for the 16-electrode system, including computation time, $t$, and image error $E_{\text {img. }}$. Explicit methods include linear back projection (LBP), Tikhonov regularization, and Tikhonov-conditioned sensitivity factor regularization (SFR). Iterative methods include algebraic reconstruction technique (ART), Landwebber iteration, and total variation regularization (TV).

\section{Optimized System Design}

The design of any real ECT system involves a basic tradeoff. While large electrodes provide greater signal-to-noise ratio, they represent integral measurements spread over a larger volume of the domain. Peng et.al. [24] suggested that in a pipe flow the axial length of electrodes should be equal to the pipe diameter to preserve the 2D approximation often made in calculating the sensitivity distributions. However, the most critical design parameter for a system is the circumferential electrode density. A system may be designed with many electrodes around the domain of interest, resulting in smaller electrodes and therefore lowered signal-to-noise ratios. Alternatively, it may be designed with fewer and 
larger electrodes, resulting in increased signal-to-noise ratio. Measured capacitance for a pair of electrodes on the far ends of the domain may be orders of magnitude smaller than for a pair of adjacent electrodes. Thus, signal-to-noise ratio deteriorates rapidly with increasing numbers of electrodes. Noisy measurements introduce false artifacts in reconstructed images. This section demonstrates a statistical investigation, identifying the optimum number of electrodes for a given domain that minimize the expected image error. The accuracy and computational ease of SFR make it an ideal method for repeatedly solving the image reconstruction problem during such an investigation. Overall image error may be analyzed as a composite of mapping error, which arises from sources other than measurement noise, and noise error due to measurement noise.

\subsection{Mapping Error}

Even without the presence of noise in the measurements, reconstructed images are subject to several factors that result in a mapping error when mapping a measurement in $\mathbf{R}^{\mathbf{m}}$ to an image vector in $\mathbf{R}^{\mathbf{n}}$. These factors are:

1. The sensitivity matrix represents a linearized system and therefore is an approximation of the true sensitivity functions.

2. The sensitivity matrix bears a large null space in $\mathbf{R}^{\mathbf{n}}$ containing many potential solutions.

3. The reconstruction algorithm may contain artificial constraints (such as regularization parameters) that are intended to compensate for other sources of error, but allow the algorithm to deliver solutions outside the null space of the sensitivity matrix.

The contribution of \#1 may only be reduced by conducting simulations of the electric field throughout the image reconstruction process in order to update $\mathbf{S}$ and account for soft-field effects, which are not considered in this work. The contribution of \#2 is reduced by increasing electrode number.

The regularization parameter, $\hat{\delta}$, for the SFR method is obtained by using the reconstruction method without measurement noise and optimizing for the value that minimizes cumulative image error for a test set of images. The test set for this exercise consisted of 20 images of random ellipsoidal regions 
occupying image fractions between $7 \%$ and $43 \%$ of the domain. Examples are shown in the left column of Figure 6. Image reconstructions conducted without any simulated measurement noise are classified as being subject only to mapping error, arising from the combined effects of the three aforementioned influencing factors.

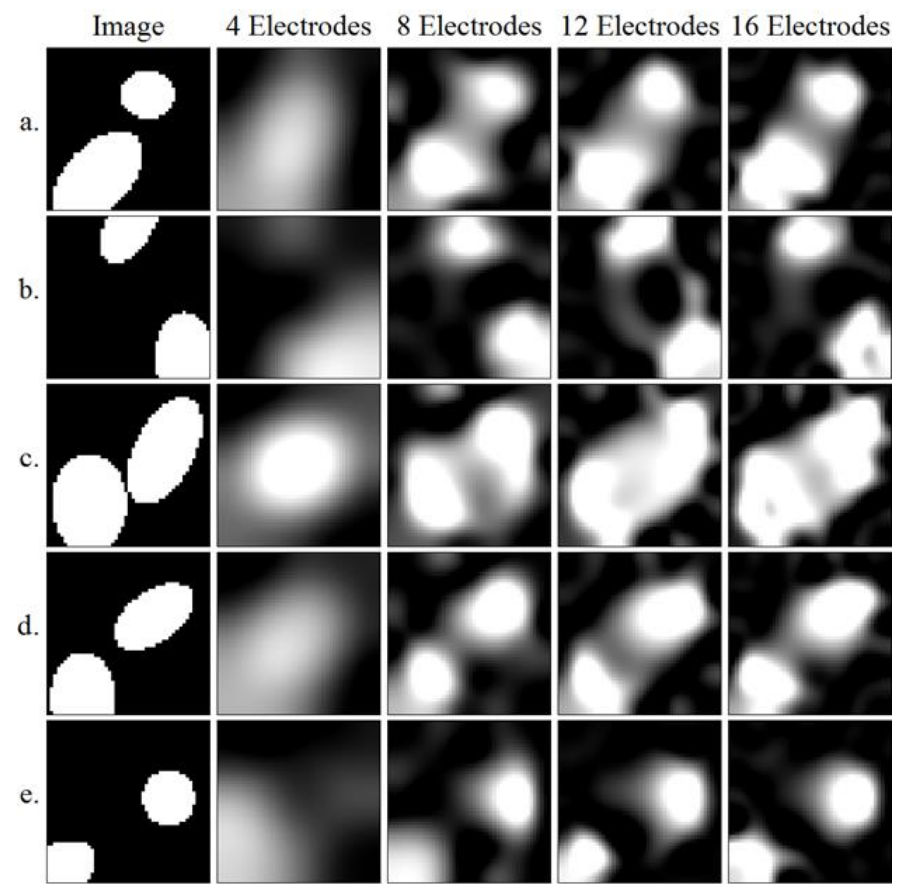

Figure 6. Image reconstructions using the SFR method subject only to mapping error for candidate tomography designs.

\subsection{Noise Error}

The second source of error is due to noise in the measurements. When noisy measurements are used, the errors in measurement values are transformed into spurious artifacts in the reconstructed image. Noise error may be isolated by comparing image reconstructions of artificially noisy measurements to reconstructions that are subject only to mapping error. Noise uncertainty of $u^{*}$ is simulated by adding a vector of random values between $-u^{*}$ and $u^{*}$ to the measurement vector $\boldsymbol{\mu}$. Image error due to noise is calculated as

$$
E_{\text {img }, \text { map }}=\left\|\boldsymbol{\xi}_{\text {clip,map }}-\boldsymbol{\xi}_{\text {clip }}\right\|_{2}
$$

where $\boldsymbol{\xi}_{\text {clip,map }}$ represents the image vector obtained when subject only to mapping error. 
Figure 7 shows mean image error over the set of 20 images due to noise. Susceptibility to noise error increases with electrode number. In Figure 8, the effects of different noise levels are shown for selected images of the test set for the 16-electrode system. Increasing noise levels are manifested in the image as false artifacts which eventually overwhelm the distinguishability of the original objects.

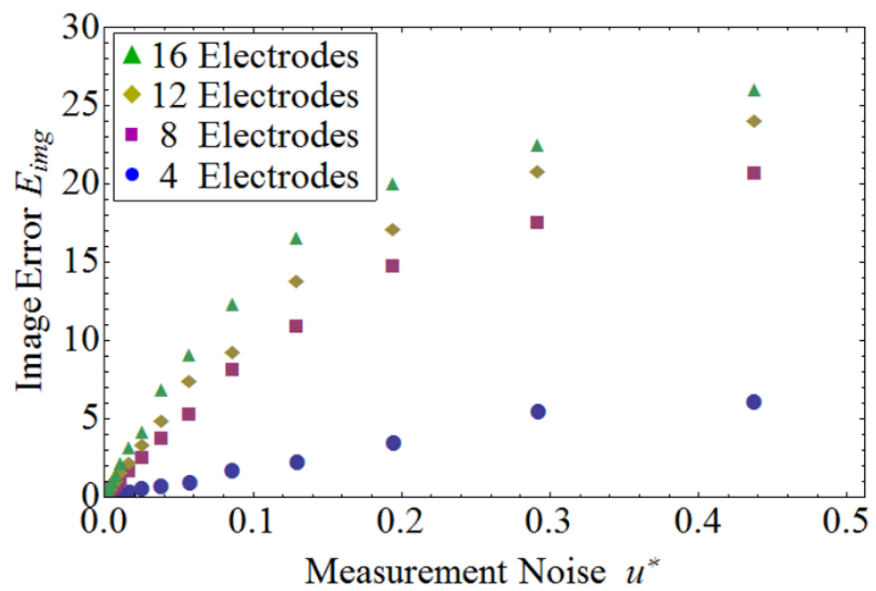

Figure 7. Mean image error over a set of 20 test images for different levels of measurement noise for the four candidate ECT designs.

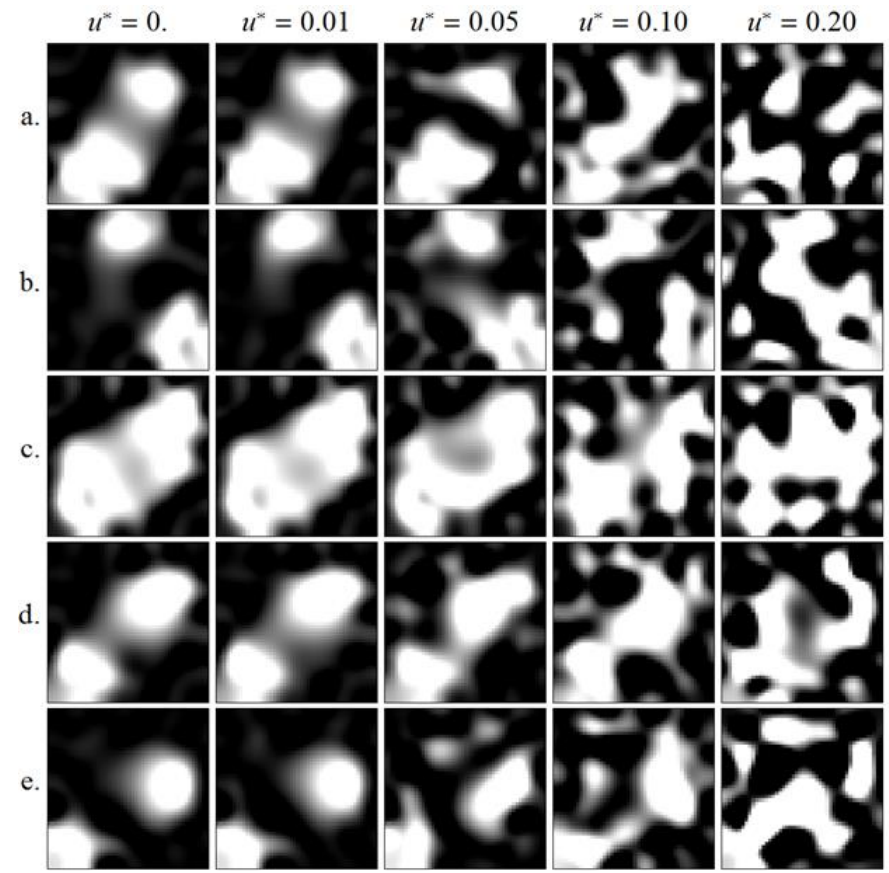

Figure 8. Image reconstructions using the SFR method for different levels of measurement noise using the 16-electrode ECT system. 


\subsection{Overall Error Minimization}

The mean overall image error over the test set is identified as the expected image error for a particular electrode design. The design may be chosen such that the expected image error is minimized. Figure 9 shows mean mapping error, noise error, and overall error for the 20 -image test set as electrode number increases. Reductions in mapping error exhibit diminishing returns with more than two electrodes per side, indicating that beyond this point, mapping error is dominated by error due to linearization of the system (factor \#1 in Section 5.1). Noise error is negligible for all systems at $u^{*}=0.01$, but outweighs mapping error for most systems at $u^{*}=0.20$. The overall image error is calculated from Equation 14 using image vectors solved with noisy measurements. It is observed that a RMS sum of mapping error and noise error is a very accurate predictor of total image error. When measurement noise is very low, the 16-electrode case performs best because mapping error is also the lowest (Figure 9a). A noise level may be identified at which the competing effects of noise and mapping error result in an optimum electrode quantity of 8 (Figure 9b). At a noise level of 0.20 , performance of the system with one electrode per side is still governed primarily by mapping error, while the other systems suffer considerably from the noise (Figure 9c). 

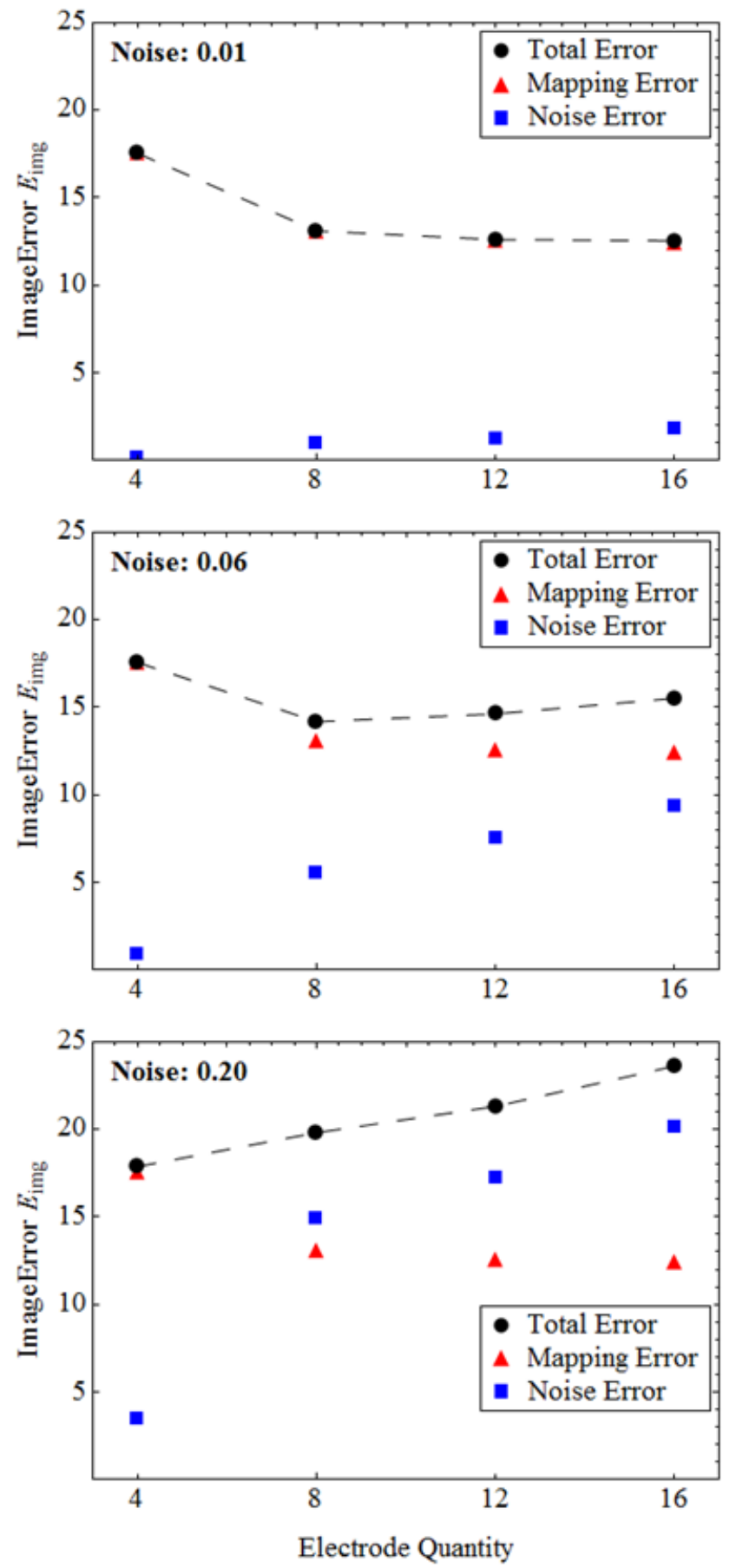

Figure 9. Mean total image error over the set of 20 test images, with contributions due to mapping error and noise error for different measurement noise levels, as a function of ECT design.

In order to validate the image error predictions of the different systems, a new set of five images is used as a validation set, which may be seen in the left column of Figure 10. Images in the set exhibit sharp features difficult for capacitance tomography systems to reconstruct accurately. It is expected that 
the mean image error values observed for the original set of test images will apply directly to the new set of validation images. Strong agreement between expected image error and mean overall image error for the validation set of five images is observed, as shown in Table 1. Image reconstructions for the validation image set are presented in Figure 10. In Figure 10, the image reconstructions using the optimal number of electrodes is used with each measurement noise level. When measurement noise is high, larger electrodes provide more accurate images than smaller electrodes, despite the lower number of measurements. However, dramatic improvements in image quality are gained when noise levels are reduced, enabling electrode size to be reduced and their number to be increased.

Table 1. Mean image error obtained for the set of validation images, compared to mean image error values predicted by the set of 20 test images.

\begin{tabular}{cccccc}
\hline Noise & & \multicolumn{4}{c}{ Electrode Number } \\
Level & & $\mathrm{K}=4$ & $\mathrm{~K}=8$ & $\mathrm{~K}=12$ & $\mathrm{~K}=16$ \\
\hline \multirow{2}{*}{0.01} & Expected & 17.5 & 13.2 & 12.6 & 12.5 \\
& Observed & 17.5 & 13.1 & 12.6 & 12.5 \\
& & & & & \\
\multirow{2}{*}{0.06} & Expected & 17.6 & 14.1 & 14.3 & 15.8 \\
& Observed & 17.5 & 14.2 & 14.6 & 15.5 \\
& & & & & \\
\multirow{2}{*}{0.20} & Expected & 18.0 & 19.5 & 22.0 & 23.7 \\
& Observed & 17.9 & 19.8 & 21.3 & 23.6 \\
\hline
\end{tabular}




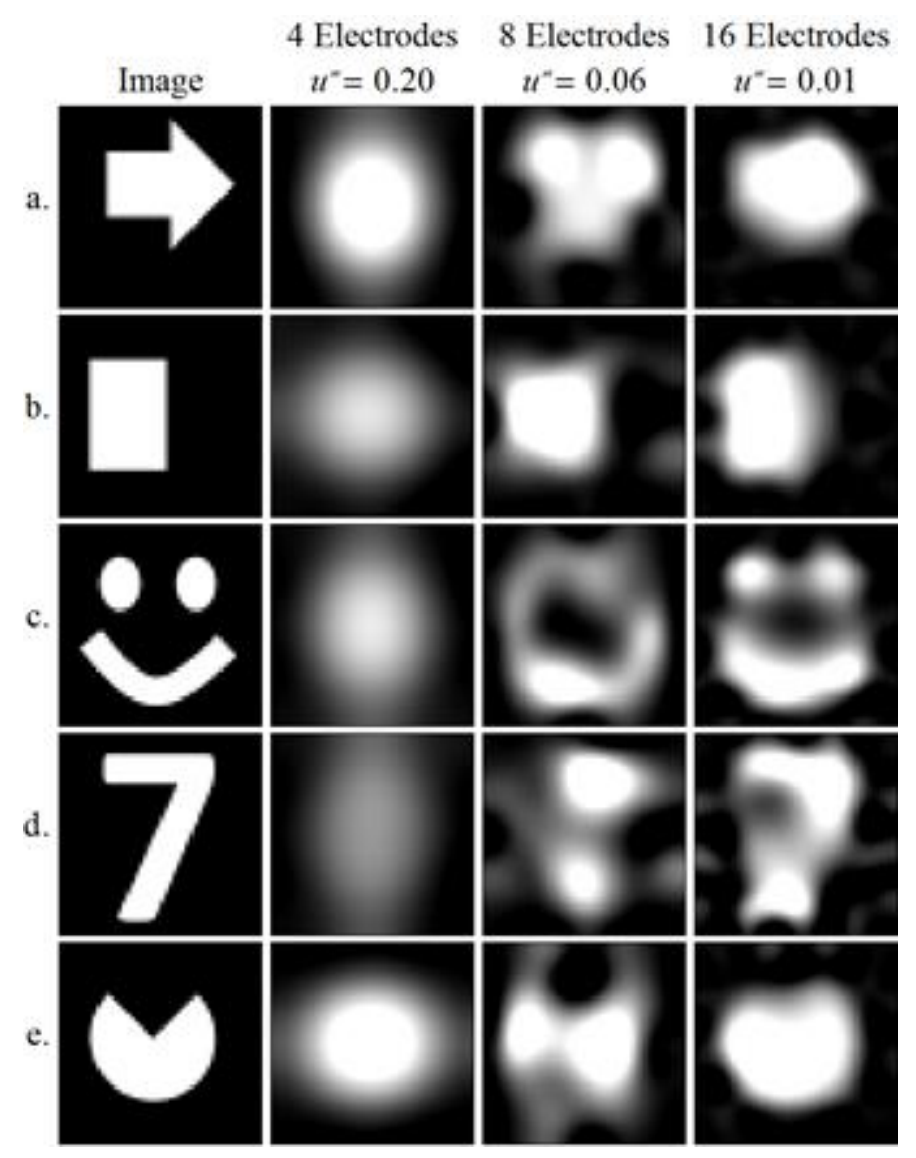

Figure 10. Image reconstructions using the SFR method for best-performing ECT design candidates at signal noise levels of $0.20,0.06$ and 0.01 .

\section{Conclusion}

The sensitivity factor method (SFR) has been developed as a new, explicit reconstruction algorithm and shown to perform better than other explicit algorithms such as linear back projection and Tikhonov regularization. The SFR solution provides an attractive alternative for applications where the need for fast image reconstruction precludes the use of iterative image reconstruction methods. The SFR method is an ideal image reconstruction technique for performing theoretical statistical investigations into design of ECT systems. As an example, a study has been presented in which the optimal number of electrodes in an ECT system is determined using SFR reconstructions of a set of test images. Error contributions due to noisy measurements and those due to approximations in mapping to the image vector 
space present competing effects that result in the existence of an optimum number of electrodes for a given level of measurement noise.

\section{Acknowledgements}

The authors gratefully recognize financial support for this work from Cooling Technologies Research Center, a National Science Foundation Industry/University Cooperative Research Center and Purdue University. Helpful technical discussions with Drs. Charles Bouman (Electrical and Computer Engineering), Gregory Buzzard (Mathematics), and Justin Weibel (Mechanical Engineering) of Purdue University are deeply appreciated. 


\section{References}

[1] H. M. Prasser, A. Bottger and J. Zschau, "A New Electrode-Mesh Tomograph for Gas-Liquid Flows," Flow Measurement and Instrumentation, vol. 9, pp. 111-119, 1998.

[2] M. J. Da Silva, S. Thiele, L. Abdulkareem, B. J. Azzopardi and U. Hampel, "High-Resolution GasOil Two-Phase Flow Visualization with a Capacitance Wire-Mesh Sensor," Flow Measurement and Instrumentation, vol. 21, pp. 191-197, 2010.

[3] C. G. Xie, S. M. Huang, B. S. Hoyle, R. Thorn, C. Lenn, D. Snowden and M. S. Beck, "Electrical Capacitance Tomography for Flow Imaging: System Model for Development of Image Reconstruction Algorithm and Design of Primary Sensors," IEE Proceedings, vol. 139, no. 1, pp. 8998, 1992.

[4] N. Reinecke and D. Mewes, "Recent Developments and Industrial Research Applications of Capacitance Tomography," Measurement Science and Technology, vol. 7, pp. 233-246, 1996.

[5] T. R. McKeen and T. S. Pugsley, "The Influence of Permittivity Models on Phantom Images Obtained from Electrical Capacitance Tomography," Measurement Science and Technology, vol. 13, pp. 1822-1830, 2002.

[6] B. Kortschak and B. Brandstatter, "A FEM-BEM Approach Using Level-Sets in Electrical Capacitance Tomography," The International Journal for Computation and Mathematics in Electrical and Electronic Engineering (COMPEL), vol. 24, no. 2, pp. 591-605, 2005.

[7] B. Kortschak, H. Wegleiter and B. Brandstatter, "Formulation of Cost Functionals for Different Measurement Principles in Nonlinear Capacitance Tomography," Measurement Science and Technology, no. 18, pp. 71-78, 2007.

[8] L. F. Zhang and H. X. Wang, "A New Normalization Method Based on Electrical Field Lines for Electrical Capacitance Tomography," Measurement Science and Technology, vol. 20, 2009.

[9] S. Teniou, M. Meribout, T. A. Hanaei, F. A. Zaabi, R. Banihashim and S. A. Ghafri, "A New Constrained Hierarchical Reconstruction Method for Electrical Capacitance Tomography," Flow Measurement and Instrumentation, vol. 23, pp. 66-75, 2012.

[10] T. Loser, R. Wajman and D. Mewes, "Electrical Capacitance Tomography: Image Reconstruction Along Electrical Field Lines," Measurement Science and Technology, vol. 12, pp. 1083-1091, 2001.

[11] W. Q. Yang and L. Peng, "Image Reconstruction Algorithms for Electrical Capacitance Tomography," Measurement Science and Technology, vol. 14, pp. R1-R13, 2003.

[12] A. M. Olmos, M. A. Carvajal, D. P. Morales, A. Garcia and A. J. Palma, "Development of an Electrical Capacitance Tomography System using Four Rotating Electrodes," Sensors and Actuators A: Physical, vol. 148, pp. 366-375, 2008.

[13] X. Y. Dong and S. Q. Guo, "Image Reconstruction Method for Electrical Capacitance Tomography based on the Combined Series and Parallel Normalization Model," Measurement Science and Technology, vol. 19, no. 4, p. 045502, 2008.

[14] S. H. Taylor and S. V. Garimella, "Shape-Energy Evolutionary Reconstruction Algorithm for Electrical Capacitance Tomography in a High-Aspect-Ratio Domain," Sensors and Actuators A: Physical, p. (in review), 2015.

[15] R. Wajman and R. Banasiak, "Tunnel-Based Method of Sensitivity Matrix Calculation for 3D-ECT Imaging," Sensor Review, vol. 34, no. 3, pp. 273-283, 2014.

[16] J. Lucas, S. Hole and C. Batis, "Simple and Direct Calculation of Capacititve Sensor Sensitivity Map," International Journal for Computation and Mathematics in Electrical and Electronic Engineering (COMPEL), vol. 27, no. 1, pp. 307-318, 2008.

[17] Y. Yang and L. Peng, "A Configurable Electrical Capacitance Tomography System Using a Combining Electrode Strategy," Measurement Science and Technology, vol. 24, 2013. 
[18] J. Lei, S. Liu, Z. Li, H. I. Schlaberg and M. Sun, "An Image Reconstruction Algorithm based on the Regularized Total Least Squares Method for Electrical Capacitance Tomography," Flow Measurement and Instrumentation, vol. 19, pp. 325-330, 2008.

[19] J. Liu, L. Lin, W. Zhang and G. Li, "A Novel Combined Regularization Algorithm of Total Variation and Tikhonov Regularization for Open Electrical Impedance Tomography," Physiological Measurement, vol. 34, pp. 823-838, 2013.

[20] H. X. Wang and L. Tang, "Total Variation Regularization used in Electrical Capacitance Tomography," in AIP Conference Proceedings. The Ultimate Measurement Challenge, Macao, China, pg 911-919., 2007.

[21] Wolfram Research Inc., Mathematica, Version 8.0, Champaign, IL, 2010.

[22] W. Stefan, "Total Variation Regularization for Linear Ill-Posed Inverse Problems: Extensions and Applications," Ph.D. Thesis Arizona State University, 2008.

[23] S. V. Venkatakrishnan, C. A. Bouman and B. Wohlberg, "Plug-and-Play Priors for Model Based Reconstruction," in IEEE Global Conference on Signal and Information Processing (GlobalSIP) pp 945-948, Austin, Texas, USA., 2013.

[24] L. Peng, C. Mou, D. Yao, B. Zhang and D. Xiao, "Determination of the Optimal Axial Length of the Electrode in an Electrical Capacitance Tomography Sensor," Flow Measurement and Instrumentation, vol. 16, pp. 169-175, 2005. 


\section{List of Tables}

Table 1. Mean image error obtained for the set of validation images, compared to mean image error values predicted by the set of 20 test images. 


\section{List of Figures}

Figure 1. Domain and mesh for candidate tomography systems with (a) 4 electrodes, (b) 8 electrodes, (c) 12 electrodes, and (d) 16 electrodes.

Figure 2. Referenced capacitance values obtained for the 8-electrode system obtained by direct simulation and by the linear approximation before the use of scaling factors, $\boldsymbol{\beta}$.

Figure 3. Selected sensitivity distributions from the 16-electrode design. Tunnels of positive sensitivity (red) reside within a background of negative sensitivity (blue).

Figure 4. Image reconstructions for the 8-electrode system using the USFR method, $\hat{\mathbf{S}}^{-1} \hat{\boldsymbol{\mu}}=\hat{\boldsymbol{\xi}}$.

Figure 5. Comparative study of image reconstructions using various explicit and iterative methods for the 16-electrode system, including computation time, $t$, and image error $E_{\text {img. }}$. Explicit methods include linear back projection (LBP), Tikhonov regularization, and Tikhonov-conditioned sensitivity factor regularization (SFR). Iterative methods include algebraic reconstruction technique (ART), Landwebber iteration, and total variation regularization (TV).

Figure 6. Image reconstructions using the SFR method subject only to mapping error for candidate tomography designs.

Figure 7. Mean image error over a set of 20 test images for different levels of measurement noise for the four candidate ECT designs.

Figure 8. Image reconstructions using the SFR method for different levels of measurement noise using the 16-electrode ECT system.

Figure 9. Mean total image error over the set of 20 test images, with contributions due to mapping error and noise error for different measurement noise levels, as a function of ECT design.

Figure 10. Image reconstructions using the SFR method for best-performing ECT design candidates at signal noise levels of $0.20,0.06$ and 0.01 . 